\title{
Erratum to: Integrity and Internal Control in Information Systems V
}

\author{
Michael Gertz \\ University of California at Davis, USA
}

\section{Erratum to: \\ M. Gertz (Ed.) \\ Integrity and Internal Control in Information Systems V \\ DOI: $10.1007 / 978-0-387-35693-8$}

The book was inadvertently published with an incorrect name of the copyright holder. The name of the copyright holder for this book is: (c) IFIP International Federation for Information Processing. The book has been updated with the changes. 